\title{
Même si le BMS continue à vous énerver en 2009, espérons que vous y trouverez aussi du plaisir
}

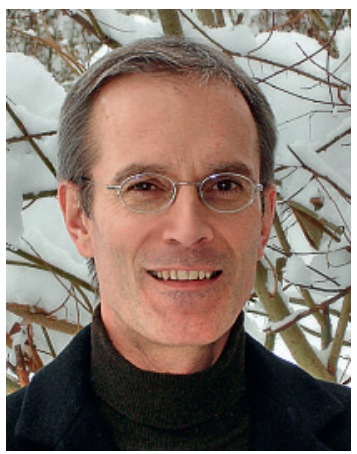

Bruno Kesseli

Il paraît qu'un jour, Willy Brandt a qualifié les journalistes de «figures marginales de l'industrie du bois», dans une riposte du tac au tac certes peu flatteuse mais sans appel envers la corporation des «plumitifs» qui, de son côté, n'a pas toujours pris des gants pour critiquer l'ancien chancelier fédéral. Puisque le Bulletin des médecins suisses continuera d'être imprimé sur du papier fait de pâte de bois avec une surface légèrement glacée, la rédaction du BMS entre également dans la catégorie de personnes définie par Willy Brand.

Néanmoins, il en va un peu différemment «chez nous» que dans la presse à laquelle Willy Brand pensait quand il a lancé sa fameuse remarque. Et comme le passage à l'an neuf donne traditionnellement lieu à des rétrospectives, perspectives, réflexions et analyses en tout genre, qu'il me soit permis de formuler à nouveau quelques considérations de base concernant le BMS et ses caractéristiques.

Il vaut la peine de souligner d'emblée que le contenu du BMS est rédigé principalement, à quelques exceptions près, non pas par des journalistes, mais par son lectorat. En conséquence, les auteurs publiés dans le BMS ne sont généralement pas concernés par la citation de Leo

\section{«Nous espérons que le BMS continuera de publier, en sa qualité de forum ouvert du corps médical, des articles intéressants, divertissants et inspirés»}

1 Leo Longanesi (1905-1957), journaliste italien, éditeur, dessinateur, illustrateur et humoriste.
Longanesi [1] supputant $\mathrm{qu}^{\prime} \ll u n$ journaliste est quelqu'un qui explique clairement à d'autres des choses qu'il ne comprend pas lui-même» ni par celle de Karl Kraus, journaliste et humoriste, qui affirmait que «ne pas avoir d'idée et pouvoir l'exprimer est le propre du journaliste».

Le BMS dispose d'un nombre très élevé d'articles potentiels. On pourrait s'imaginer que la rédaction utilise cette situation confortable pour prendre ses aises et puiser plus ou moins par hasard dans cette réserve fort bien garnie. Le contraire est plus proche de la réalité. Abstraction faite de la première partie des citations sus- mentionnées, donc des qualités journalistiques dont manquent parfois les manuscrits que nous recevons, leur qualité et hétérogénéité représentent un énorme défi pour la rédaction. En votre qualité de lectrice ou de lecteur, vous pouvez être assuré-e que nous prenons très au sérieux l'exigence du BMS de refléter la diversité du corps médical suisse le plus justement possible. L'élargissement de la rédaction effectué il y a un an garantit que les décisions relatives aux articles à publier sont prises sur la base d'un large consensus, et je tiens ici à remercier cordialement mes collègues de la rédaction pour leur précieux travail.

Mais les raisons de s'énerver au sujet du BMS ne manqueront pas non plus à l'avenir, malgré les efforts de la rédaction. En raison de la situation économique devenue plus difficile, nous avons dû réduire sensiblement le nombre de pages rédactionnelles par édition pour économiser des coûts de production. Nous devons ainsi exiger des auteurs plus de discipline concernant le respect de la longueur prescrite. Par ailleurs, le délai de parution des articles est devenu plus long et notre taux de refus d'articles a légèrement augmenté tout en demeurant malgré tout très bas par rapport à d'autres revues professionnelles.

Naturellement, la rédaction se trompe aussi parfois, mais dans le fond, la question de l'insatisfaction des lecteurs fait déjà partie de son concept. On s'énerve sur les injustices économiques, l'arrogance des autorités, les collègues dogmatiques ou romanesques, les passages à vide des institutions ou les politiciens «incapables». Tant que toute la diversité du paysage sanitaire suisse pourra se refléter dans le BMS, sa lecture hebdomadaire restera source de menues irritations.

Rien de tragique car nous espérons que le BMS continuera de publier, en sa qualité de forum ouvert du corps médical, des articles intéressants, divertissants et inspirés. Les nombreux échos émanant de notre lectorat attestent que nous remplissons cette fonction et nous motivent à viser la quadrature du cercle: publier une revue suscitant l'intérêt de tous les médecins établis en Suisse.

Au nom de la rédaction, je vous souhaite une belle et heureuse année 2009.

Bruno Kesseli, rédacteur en chef 